\title{
Nanofibre Fabrication by Femtosecond Laser Ablation of Silica Glass
}

Krishnan Venkatakrishnan

Ryerson University

\section{Dheeraj Vipparty}

Ryerson University

\section{Bo Tan}

Ryerson University

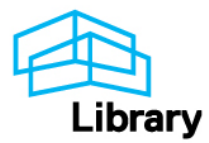




\title{
Nanofibre fabrication by femtosecond laser ablation of silica glass
}

\author{
Krishnan Venkatakrishnan, ${ }^{1}$ Dheeraj Vipparty, ${ }^{2}$ \\ and Bo $\operatorname{Tan}^{2, *}$ \\ ${ }^{I}$ Department of Mechanical \& Industrial Engineering, Ryerson University, Toronto, ON, Canada \\ ${ }^{2}$ Department of Aerospace Engineering, Ryerson University, Toronto, ON, Canada \\ *tanbo@ryerson.ca
}

\begin{abstract}
This article presents a fabrication technique for generating densely populated and randomly oriented silica nanofibres by direct ablation of silica glass using a femtosecond laser with $12.4 \mathrm{MHz}$ repetition rate and a pulse width of $214 \mathrm{fs}$, under ambient conditions. Four types of nanofibres with diameters ranging from a few tens of nanometers to a few hundreds of nanometers were formed. Some fibers reach lengths of $10 \mathrm{~mm}$. The possible mechanisms for fibre formation have been explored.
\end{abstract}

(C)2011 Optical Society of America

OCIS codes: (140.3390) Laser materials processing; (140.3510) Lasers, fiber; (220.4241) Nanostructure fabrication; (140.7090) Ultrafast lasers; (120.6810) Thermal effects.

\section{References and links}

1. L. Tong, and E. Mazur, "Glass nanofibers for micro- and nano-scale photonic devices," J. Non-Cryst. Solids 354(12-13), 1240-1244 (2008).

2. Z. M. Huang, Y. Z. Zhang, M. Kotaki, and S. Ramakrishna, "A review on polymer nanofibers by electrospinning and their applications in nanocomposites," Compos. Sci. Technol. 63(15), 2223-2253 (2003).

3. S. Ramakrishna, An introduction to electrospinning and nanofibers (World Scientific Pub Co Inc, 2005).

4. L. Tong, R. R. Gattass, J. B. Ashcom, S. He, J. Lou, M. Shen, I. Maxwell, and E. Mazur, "Subwavelengthdiameter silica wires for low-loss optical wave guiding," Nature 426(6968), 816-819 (2003).

5. S. S. Choi, S. G. Lee, S. S. Im, S. H. Kim, and Y. L. Joo, "Silica nanofibers from electrospinning/sol-gel process," J. Mater. Sci. Lett. 22(12), 891-893 (2003).

6. C. Yan, T. Zhang, and P. S. Lee, "Flow assisted synthesis of highly ordered silica nanowire arrays," Appl. Phys., A Mater. Sci. Process. 94(4), 763-766 (2009).

7. L. Dai, L. You, X. Duan, W. Lian, and G. Qin, "Growth of silica nanowire arrays by reaction of Si substrate with oxygen using Ga as catalyst," Phys. Lett. A 335(4), 304-309 (2005).

8. Z. W. Pan, Z. R. Dai, C. Ma, and Z. L. Wang, "Molten gallium as a catalyst for the large-scale growth of highly aligned silica nanowires," J. Am. Chem. Soc. 124(8), 1817-1822 (2002).

9. Z. Wang, R. Gao, J. Gole, and J. Stout, "Silica nanotubes and nanofiber arrays," Adv. Mater. 12(24), 1938-1940 (2000).

10. D. Yu, Q. L. Hang, Y. Ding, H. Zhang, Z. Bai, J. Wang, Y. Zou, W. Qian, G. Xiong, and S. Feng, “Amorphous silica nanowires: Intensive blue light emitters,” Appl. Phys. Lett. 73(21), 3076-3078 (1998).

11. X. Wu, W. Song, K. Wang, T. Hu, B. Zhao, Y. Sun, and J. Du, "Preparation and photoluminescence properties of amorphous silica nanowires," Chem. Phys. Lett. 336(1-2), 53-56 (2001).

12. L. Tong, J. Lou, Z. Ye, G. T. Svacha, and E. Mazur, "Self-modulated taper drawing of silica nanowires," Nanotechnology 16(9), 1445-1448 (2005).

13. V. N. Tokarev, S. Lazare, C. Belin, and D. Debarre, "Viscous flow and ablation pressure phenomena in nanosecond UV laser irradiation of polymers," Appl. Phys., A Mater. Sci. Process. 79(4-6), 717-720 (2004).

14. G. A. J. Markillie, H. J. Baker, F. J. Villarreal, and D. R. Hall, "Effect of vaporization and melt ejection on laser machining of silica glass micro-optical components," Appl. Opt. 41(27), 5660-5667 (2002).

15. A. Zoubir, L. Shah, K. Richardson, and M. Richardson, "Practical uses of femtosecond laser micro-materials processing," Appl. Phys., A Mater. Sci. Process. 77, 311-315 (2003).

16. E. Gamaly, A. Rode, B. Luther-Davies, and V. Tikhonchuk, "Ablation of solids by femtosecond lasers: Ablation mechanism and ablation thresholds for metals and dielectrics," Phys. Plasmas 9(3), 949 (2002).

17. V. Koubassov, J. Laprise, F. Théberge, E. Förster, R. Sauerbrey, B. Müller, U. Glatzel, and S. Chin, "Ultrafast laser-induced melting of glass," Appl. Phys., A Mater. Sci. Process. 79, 499-505 (2004).

18. R. E. Russo, X. Mao, J. J. Gonzalez, and S. S. Mao, "Femtosecond laser ablation ICP-MS," J. Anal. At. Spectrom. 17, 1072-1075 (2002).

19. M. Feit, A. Komashko, and A. Rubenchik, "Ultra-short pulse laser interaction with transparent dielectrics," Appl. Phys., A Mater. Sci. Process. 79(7), 1657-1661 (2004).

20. S. Chin, "From multiphoton to tunnel ionization," Advances in multi-photon processes and spectroscopy, S. H. Lin, A. A. Villaeys and Y. Fujimura, eds. World Scientific, Singapore, 16, 249-272 (2004).

\#145518 - \$15.00 USD Received 7 Apr 2011; revised 30 May 2011; accepted 30 May 2011; published 3 Aug 2011

(C) 2011 OSA

15 August 2011/ Vol. 19, No. 17 / OPTICS EXPRESS 15770 
21. A. Kaiser, B. Rethfeld, M. Vicanek, and G. Simon, "Microscopic processes in dielectrics under irradiation by subpicosecond laser pulses," Phys. Rev. B 61(17), 11437-11450 (2000).

22. A. Brodeur, and S. Chin, "Band-gap dependence of the ultrafast white-light continuum," Phys. Rev. Lett. 80(20), 4406-4409 (1998).

23. F. Korte, J. Koch, and B. Chichkov, "Formation of microbumps and nanojets on gold targets by femtosecond laser pulses," Appl. Phys., A Mater. Sci. Process. 79(4-6), 879-881 (2004).

24. M. R. Kasaai, V. Kacham, F. Theberge, and S. L. Chin, "The interaction of femtosecond and nanosecond laser pulses with the surface of glass," J. Non-Cryst. Solids 319(1-2), 129-135 (2003).

25. T. Tamaki, W. Watanabe, and K. Itoh, "Laser micro-welding of transparent materials by a localized heat accumulation effect using a femtosecond fiber laser at $1558 \mathrm{~nm}$," Opt. Express 14(22), 10460-10468 (2006).

26. B. Tan, and K. Venkatakrishnan, "Synthesis of fibrous nanoparticle aggregates by femtosecond laser ablation in air," Opt. Express 17(2), 1064-1069 (2009).

27. A. Ben-Yakar, R. L. Byer, A. Harkin, J. Ashmore, H. A. Stone, M. Shen, and E. Mazur, "Morphology of femtosecond-laser-ablated borosilicate glass surfaces," Appl. Phys. Lett. 83(15), 3030-3032 (2003).

28. K. L. Wray, and T. J. Connolly, "Thermal conductivity of clear fused silica at high temperatures," J. Appl. Phys. 30(11), 1702-1705 (1959)

29. B. Luther-Davies, A. V. Rode, N. R. Madsen, and E. G. Gamaly, "Picosecond high-repetition-rate pulsed laser ablation of dielectrics: The effect of energy accumulation between pulses," Opt. Eng. 44(5), 051102-051108 (2005).

30. A. Ben-Yakar, A. Harkin, J. Ashmore, R. L. Byer, and H. A. Stone, "Thermal and fluid processes of a thin melt zone during femtosecond laser ablation of glass: The formation of rims by single laser pulses,” J. Phys. D 40(5), 1447-1459 (2007)

31. S. Juodkazis, H. Misawa, O. A. Louchev, and K. Kitamura, "Femtosecond laser ablation of chalcogenide glass: Explosive formation of nano-fibres against thermo-capillary growth of micro-spheres," Nanotechnology 17(19), 4802-4805 (2006).

\section{Introduction}

Nanofibres are simple one-dimensional nanostructures that received much attention in the field of research and development during recent years due to their unique properties and potential applications in various areas. The sub-wavelength nanofibres generated from glass can be utilized as waveguides for optical communication, and as sensors [1]. Manipulation of these fibres into an assembly of various patterns or woven networks for filtration purposes is also possible as the fibres display high mechanical strength and pliability [1,2]. There have been several techniques reported to produce nanofibres such as: drawing, template synthesis, phase separation, self-assembly, and electrospinning $[2,3]$. Tong et al. have employed a twostep drawing process for fabricating long uniform silica nanowires using a flame-heated drawing method. The process allowed for the drawing of a single strand of aligned nanowire which had diameters ranging from 50 to $1100 \mathrm{~nm}$ and lengths up to tens of millimeters, with minimum irregularities along the surface [4]. Electrospinning is another widely used technique to generate silica nanofibres. This multiple-step process involves (complicated) gelpreparation, spinning and annealing. In a study conducted by Choi et al., silica nanofibres were obtained by employing electrospinning and a sol-gel method. The acquired nanofibres were randomly oriented with diameters ranging from less than $200 \mathrm{~nm}$ to more than $1000 \mathrm{~nm}$ [5].

To synthesize nanofibres with diameters below $100 \mathrm{~nm}$, various approaches have been adopted by research teams [6-9]. Yu et al. [10] reported a method that can produce large-scale silica cylindrical nanowires with a diameter distribution of around $15 \mathrm{~nm}$. However, this process requires substantial sample preparation as it involves sintering of silicon, silica and $\mathrm{Fe}$ powder, followed by baking of the sample at $1200{ }^{\circ} \mathrm{C}$ for 20 hours. Wu et al. [11] suggested the carbothermal reduction process than can fabricate a fibre bunch with individual fibres having diameters around $60 \mathrm{~nm}$. The process involves ball milling of the mixtures of $\mathrm{SiO}_{2}$, $\mathrm{Fe}\left(\mathrm{NO}_{3}\right)_{3} .9 \mathrm{H} 2 \mathrm{O}$ and active carbons, for 20 hours in ethanol media. The mixture is pressed under 10Mpa pressure and calcined at $1350{ }^{\circ} \mathrm{C}$ for another 3 hours in flowing argon. The proposed procedure takes up a considerable amount of time and exhibits a certain degree of complexity in sample preparation. The self-modulated taper drawing technique demonstrated by Tong et al. [12] can generate a continuous silica nanowire with diameters as low as $20 \mathrm{~nm}$. Nanowires obtained via this approach exhibit excellent diameter uniformity and intrinsic surface smoothness, but the process calls for a high degree precision and is susceptible to any minor fluctuations which can create abrupt taper or breakage of the wire. 
Recent publications on pulsed laser ablation of dielectric-sample state that nanosecond [13] and microsecond lasers [14] are capable of producing fibres with diameters in nanometer $(\sim 150 \mathrm{~nm})$ and micrometer respectively. Furthermore, the fibres attained have a maximum length of $1 \mathrm{~mm}$. However, to the best of our knowledge, the use of femtosecond laser to produce nanofibres with nanometer diameters has not been reported. In this article, we propose a simple, single-step technique which requires minimal sample preparation and short processing time to produce dense nanofibres of silica glass in the form of intertwined randomly oriented structures. By irradiating silica glasses surface with a femtosecond laser, densely crowded fibres have been obtained with dimension ranging from tens of nanometers to a few hundreds of nanometers for thickness, and up to several millimeters for lengths.

Femtosecond laser ablation is a non-contact micromachining technique which facilitates surface patterning of materials with minimal thermal substrate diffusion. Femtosecond lasers have the capacity to transfer energy into the target material in a very short interval of time, thus concentrating thermal energy in and around the focal region shunning thermal diffusion [15]. The peak intensity of a femtosecond laser can reach to the order of $\mathrm{GW} / \mathrm{cm}^{2}$ or TW/ $/ \mathrm{cm}^{2}$ [16]. Therefore, the femtosecond laser can process materials with high energy bandgap (dielectrics). As the femtosecond laser pulses interact with the substrate, the dielectric material undergoes melting [17] due to heat accumulation. High repetition rate and the explosive recoil pressure from the shock waves [18] significantly influence mass material removal leading to the formation of nanostructures over the sample surface. The purpose of this report is to put forward a manufacturing technique which can produce nanofibres of varying diameters and lengths on a large scale suitable for industrial environment. Efforts have also been made to shed light on possible mechanisms that promote fibre growth on a silica glass surface when the surface is irradiated with a femtosecond laser.

\section{Experimental methods}

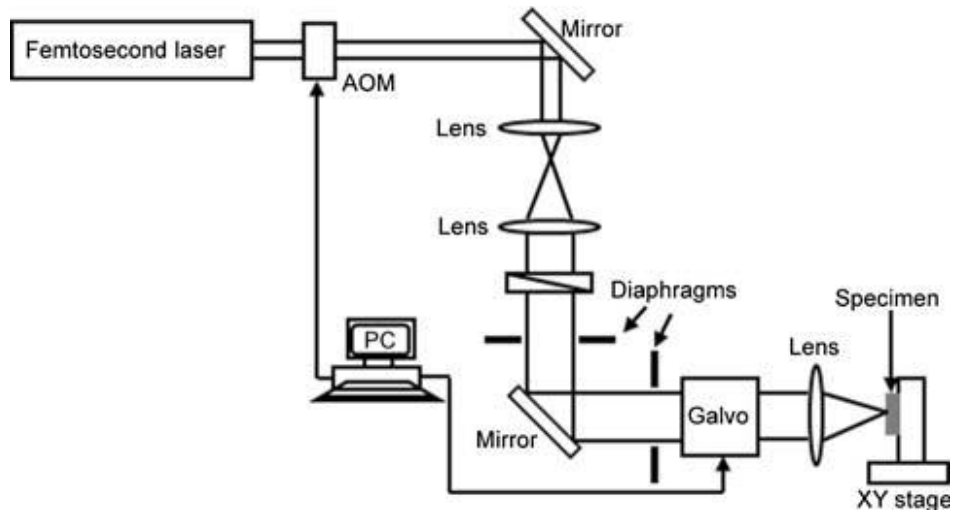

Fig. 1. Experimental setup. AOM-Acousto-optic modulator

A direct-diode pumped Yb-doped fibre amplified ultrafast laser system $(\lambda=1,030 \mathrm{~nm})$ capable of delivering a maximum output average power of $15 \mathrm{~W}$ at a pulse repetition rate ranging from $200 \mathrm{kHz}$ to $26 \mathrm{MHz}$ is employed in this experiment. In the present case, arrays of microholes were drilled on silica glass specimens using laser irradiation with a repetition rate of $12.6 \mathrm{MHz}$ and pulse width $214 \mathrm{fs}$ and dwell times in excess of $3 \mathrm{~ms}$. The experimental setup used is presented in Fig. 1. The laser beam is focused on the substrate surface and scanned using a computer controlled $\mathrm{X}$-Y axis galvanometer. The specimens are processed in air. After irradiation, the samples were examined by Scanning electron microscope (SEM) and Transmission electron microscope (TEM). 


\section{Results and discussion}

\section{$3.1 \mathrm{MHz}$ Laser pulses irradiation of dielectric material}

The femtosecond laser interaction with transparent dielectric material is a nonlinear absorption process which begins with the formation of free electron cloud in the conduction band within the solid. Ionization process such as: multiphoton ionization, followed by inverse bremsstrahlung and avalanche ionization play a major role in the production of free-electron cloud [19-22]. The free electron density, upon reaching a critical value triggers the material breakdown process. As the electron subsystem attains thermal equilibrium and undergoes rapid electron-phonon relaxation, energy is transferred into the lattice in the order of few picoseconds [23]. A fraction of this heat energy diffuses into the substrate. But with multiple pulses striking the same focal region at high repetition rate, heat accumulation becomes profound as the time interval between the pulses is less than the time required for the absorbed energy to diffuse out of the focal volume [23-25]. A finite-difference thermal diffusion model developed by Eaton et al predicts that at $\mathrm{MHz}$ repetition rate, the temperatures of the focal volume can elevate beyond $3000^{\circ} \mathrm{C}$ due to cumulative heating. At such high temperatures the viscosity of the molten material is drastically reduced, thereby making it very mobile. Therefore, cumulative heating which leads to a high material temperature plays a vital role during ablation as it (locally) melts the glass surface and enables the ablative forces to restructure the molten material into nanofibres.
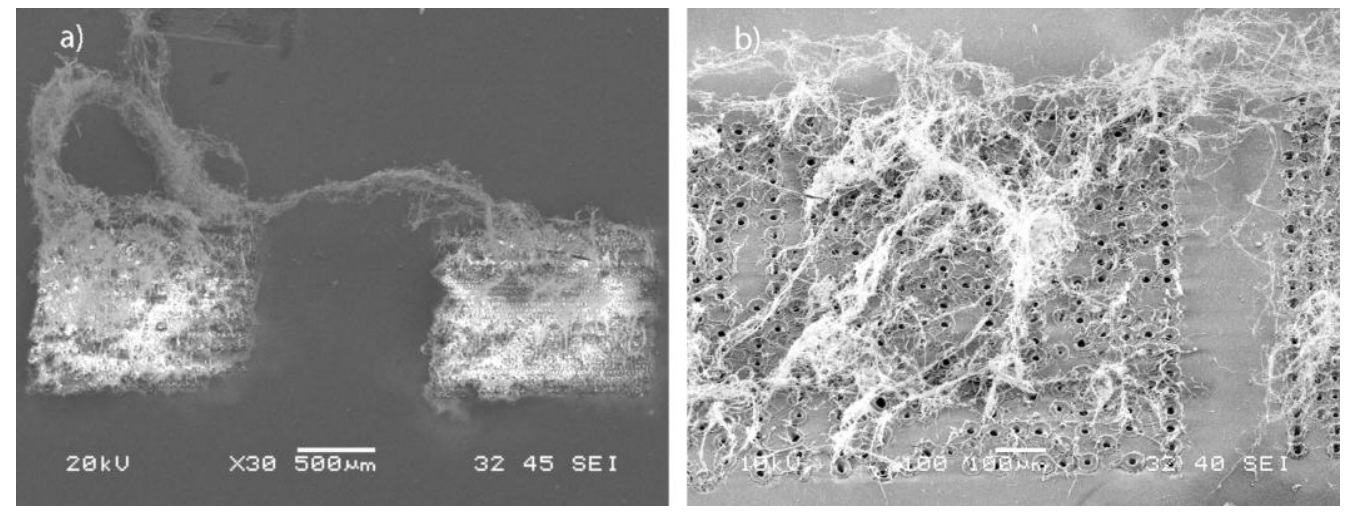

Fig. 2. a) SEM images of Silica Nanofibres generated at $12.4 \mathrm{MHz}$ and dwell time $5 \mathrm{~ms}$; b) Densely populated nanofibres emerging out around the microholes produced by femtosecond laser.

The SEM images shown in Fig. 2 illustrate the bundled up mass of nanofibres generated on silica glass surface after femtosecond laser ablation. The fibres within the mass are intertwined and randomly oriented over the substrate surface. Several thousand magnifications (via SEM and TEM) of this mass (Fig. 3, Fig. 4) reveals fibers with varying morphologies and diameter distribution. Furthermore, certain fibers attain lengths up to 10 millimeters. The mixture of fibers with different structural characteristics were formed by focusing the laser beam to a spot size of about $10 \mu \mathrm{m}$ at an average laser fluence of $1.17 \mathrm{~J} / \mathrm{cm}^{2}$ and pulse energy $922.2 \mathrm{~nJ}$. The surface damage threshold fluence of silica was determined experimentally and a fluence value of $0.93 \mathrm{~J} / \mathrm{cm}^{2}$ was obtained. The laser fluence $\left(1.17 \mathrm{~J} / \mathrm{cm}^{2}\right)$ used in our experiments was well over the damage fluence of silica glass. We believe that there could be more than one mechanism occurring simultaneously or consecutively in the background to produce fibres of different dimensions and morphologies during laser-material interaction. Henceforth, the nanofibres are segregated into different types based on morphology, length and diameter distribution. 


\subsection{Morphology of nanofibres}

Type 1 fibres (Fig. 2b) are the longest with lengths from $7 \mathrm{~mm}$ and above and with diameter in few hundreds of nanometers. Type 2 fibres display distinct spherical bead like structures attached along the fibre length (Fig. 3b). Type 3 fibres possess dimensions similar to that of Type 1 nanofibres, but have a broadened spherical structure at one end with gradual tapering to the other end (Fig. 3c). Type 4 (Fig. 3d) fibres have their diameters in few tens of nanometers and lengths up to hundreds of microns
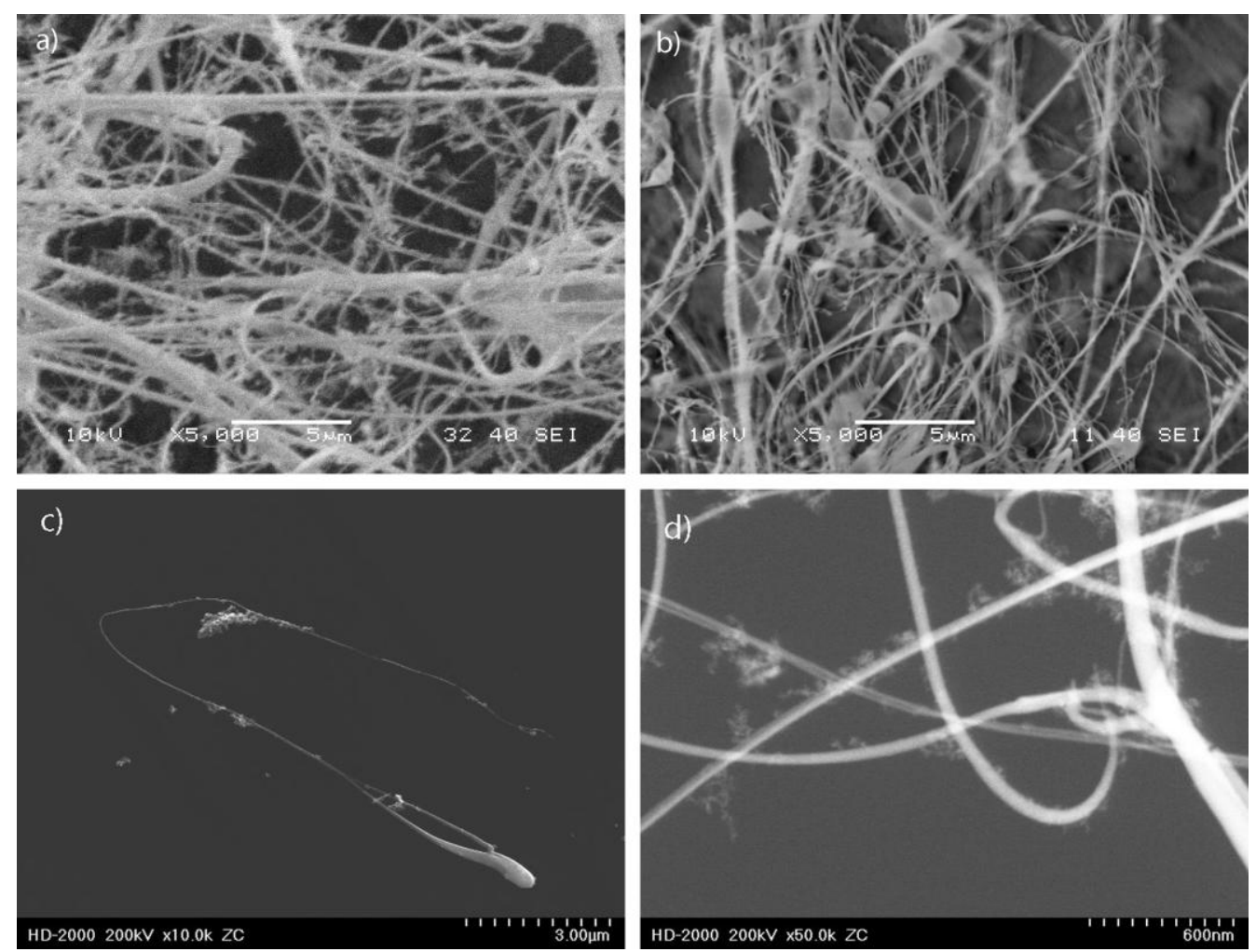

Fig. 3. Nanofibres of different morphologies a) SEM image of nanofibres with varying diameters and lengths; b) Nanofiber with spherical beads attached; c) TEM image illustrating an expelled droplet transformed into a fibre; d) TEM images silica fibres with nanometer dimensions

Further examination of the TEM micrographs (Fig. 4) show the evidence of deposited nanoparticles on the fibre surface. Nanoparticles formation can be explained as the effect of vapor condensation in the rarified plasma. The high temperature of the particles allows the particles to agglomerate (Fig. 4b) and attach to the fibre surface [26]. 

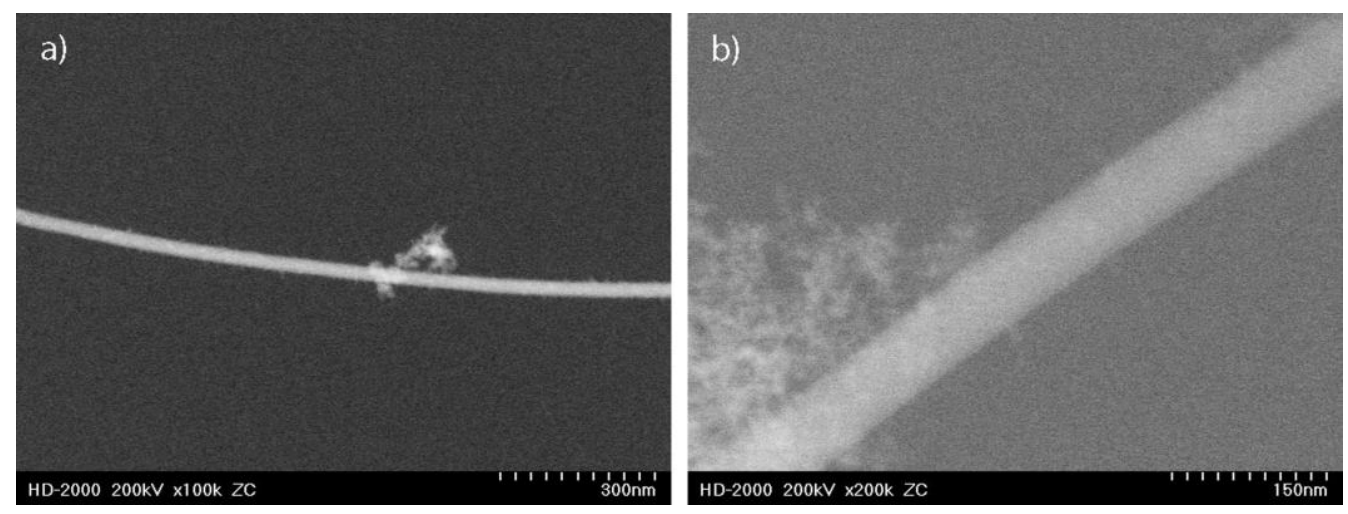

Fig. 4. TEM image of a nanofibre with diameter less than: a) $50 \mathrm{~nm}$; b) $100 \mathrm{~nm}$

\subsection{Mechanisms}

a)
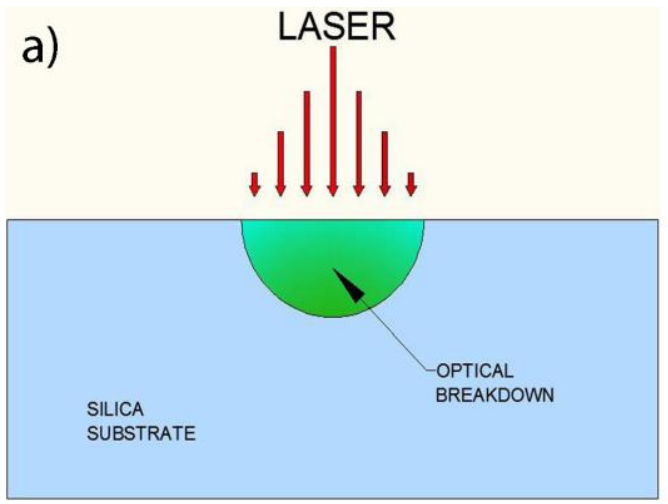

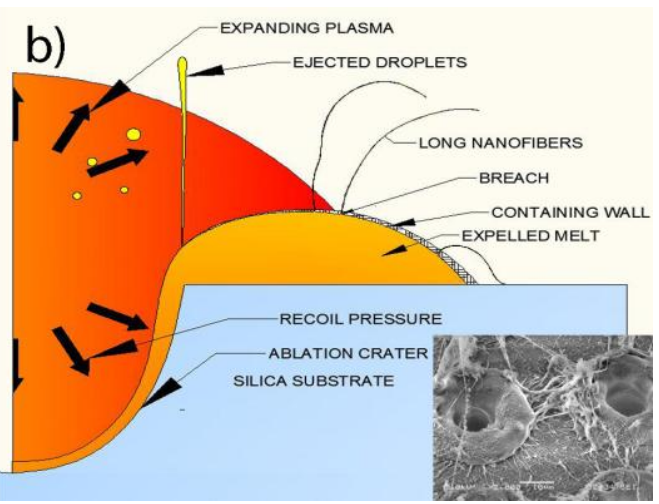

Fig. 5. a) Optical Breakdown of laser irradiated surface, b) Nanofibre formation process, inset image shows the rim created as result of melt expulsion. Fiber formation during laser ablation is predominantly via melt expulsion followed by generation of molten jets.

The process that explains the Type 1 fibre formation begins with laser pulses striking the surface of substrate (Fig. 5a) to induce plasma and commence the ablation process that gradually alters the surface morphology. Laser radiation energy is nonlinearly absorbed and transferred to the lattice within few picoseconds, after which heat diffusion into material begins [27]. The thermal conductivity of Silica at $1826.85^{\circ} \mathrm{C}$ is $2.3 \mathrm{~W} / \mathrm{m}^{\circ} \mathrm{K}$ [28]. Due to such poor thermal conductivity, significant percentage of laser energy accumulates as heat in the laser irradiated spot with every incoming pulse; consequently, the surface temperature progressively rises in a stepwise fashion [29]. The temperature continues to grow and begins to melt the material creating a molten pool under the expanding plasma. The molten pool is sustained with the aid of high pulse repetition rate. As the pulse separation time $(0.079 \mu \mathrm{s})$ is much smaller than melt time of glass $(0.4 \mu \mathrm{s}$ to $0.8 \mu \mathrm{s})$ [27]; resolidification of molten material is avoided throughout the irradiation process. The temperature of the molten pool escalates with every inbound pulse; concurrently, the viscosity of the melt reduces progressively making the melt more mobile. Eventually the melt attains viscosity similar to that of a highly mobile liquid at temperatures greater than $3000^{\circ} \mathrm{C}$. Under the influence of recoil pressure from the expanding plasma [14] the melt is driven to the edge of the crater where the colder environment surrounding the plasma impedes any further movement of the melt causing it to be deposited as distinct raised rims [30], few micrometers above the surface (Fig. 5b). The expelled material immediately begins to solidify creating a fine layer of hardened melt which acts as a containing wall (Fig. 5b). The (recently created) molten material continues to be pushed against the containing wall and is ultimately brought under 
pressure by the ablation forces. The strong temperature gradients induced by the femtosecond laser due to its Gaussian energy distribution, the solidification process of the molten material can be considered non-uniform which leads to the "weak point" areas in the containing wall. The explosive fibre ejection process then can be associated with a breach in the containing wall at the "weak point" [14], causing the hot melt to extrude out as jets. The jet persists as long as melt reservoir is available or the breach seals up. These jets originate at numerous sites over the expelled rims and as they undergo rapid cooling, alter into densely crowded nanofibres (Fig. 2b). The above mentioned process could repeat itself multiple times until the expanding crater reaches a final size and the fibres formed prior might get detached and the roots of the fibres melted back into the pool as the melt rim advances.

A cluster within the produced fibres display distinct spherical bead like structures attached along the length (Type 2, Fig. 3b) of the fibre, and can be explained as a consequence of surface destabilization due to laser induced temperature non-uniformity while cooling down and the surface tension minimization of surface area which leads to constricting of jet radius to form beads [31].

The silica glass when exposed to femtosecond laser multi-pulse irradiation, rapid heating of specimen occurs causing the glass to melt and the evolving plasma exerts recoil pressure to instigate an expulsion of melt droplets. As the droplets fly out, the volume of the droplet shrinks while leaving a liquid trail behind it. The liquid droplet upon losing momentum cools down and solidifies into a nanofibre (Type 3, Fig. 3c).

We believe that the growth process for Type 4 fibres could be similar to the one proposed by Tokarev et al [13]. He and his team fabricated nanofibres in PMMA polymer using nanosecond pulsed laser and suggested that the fibre generation is triggered by the formation of energetic droplets within the molten pool created during ablation which then propagate to the periphery of the laser spot due to pressure gradients in the molten liquid and act as heads for the viscous liquid to eject out. The ejected liquid undergoes rapid cooling and is solidified as fibres.

\section{Conclusion}

A novel method to generate silica nanofibres using femtosecond laser has been presented. This facile, single-step method provides an alternative for the production of nanofibre and sub-micron fibres in large scale with short processing time and the procedure can be carried out in air at ambient conditions. The acquired nanofibres have wide range of diameters. Fibres with thickness below $100 \mathrm{~nm}$ were obtained with length reaching up to few hundreds of microns. Based on morphology, fibers were classified into different types and the associated possible mechanisms that transpire have been elucidated The predominant process that initiates the fibre growth begins with melting of the substrate, followed by explosion expulsion of the melt by recoil pressure exerted by the expanding plume. The rapid cooling of the expelled material ultimately leads to the formation of nanofibres of extremely high aspect ratio. Study of individual fibre is quite challenging as their orientation is very random and tangled up. In this regard, ways to align the fibre array and to isolate a fibre are being extensively explored to carry out a characterization study using Atomic Force Microscopy.

\section{Acknowledgments}

This research is funded by Natural Science and Engineering Research Council of Canada.

\#145518 - \$15.00 USD Received 7 Apr 2011; revised 30 May 2011; accepted 30 May 2011; published 3 Aug 2011

(C) 2011 OSA

15 August 2011 / Vol. 19, No. 17 / OPTICS EXPRESS 15776 